\title{
Faculty Development Advancements-Lessons Learned in a Time of Change
}

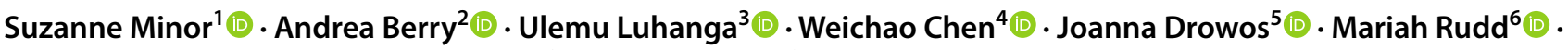 \\ Victoria S. Kaprielian ${ }^{7}$ (D) Jean M. Bailey ${ }^{8}$ (i) $\cdot$ Shanu Gupta ${ }^{9}(\mathbb{C}$
}

Accepted: 9 February 2022 / Published online: 24 February 2022

(c) The Author(s) under exclusive licence to International Association of Medical Science Educators 2022

\begin{abstract}
Faculty development (FD) activities at colleges of medicine shifted to virtual in March 2020 as the coronavirus pandemic limited in-person engagement. Medical schools delivered quality virtual faculty development (VFD) through accessing national and international experts virtually, improving faculty access to FD through recorded sessions, collaborating across institutions, and building on previous success as comfort with virtual platforms grew. Disruptive innovation and Keller's ARCS model, highlighting motivational concepts of attention, relevance, confidence, and satisfaction, guided nine faculty developers' reflections towards continuous quality improvement of VFD offerings. The convenience and low-cost availability of virtual activities mean this format will likely persist.
\end{abstract}

Keywords Faculty $\cdot$ Faculty development $\cdot$ Virtual

\section{Introduction}

Ongoing faculty development (FD) supporting educators' varied roles is required for medical school accreditation by the Liaison Committee on Medical Education [1]. FD is defined as "all activities health professionals pursue to improve their knowledge, skills, and behaviors as teachers and educators, leaders and managers, and researchers and scholars, in both individual and group settings." [2] FD often includes instructional workshops promoting educational best practices, scholarship, peer-review, promotion preparation, and leadership skills. FD is also achieved through committee work, networking events associated with instructional events, and mentoring.

As in many schools, the nine institutions of the authors who contributed to this paper have traditionally relied heavily on face-to-face FD sessions. Faculty attending these sessions valued in-person FD opportunities as evidenced by session evaluations. Faculty developers offered varied sessions useful to everyone, from junior faculty requiring specific skill development to the master teacher brushing up or becoming engaged with a teaching community of practice. Yet, participation often consisted of faculty who tended to be the most active, engaged teachers and least in need of
Suzanne Minor

seminor@fiu.edu

1 Department of Humanities, Health and Society, Florida International University Herbert Wertheim University College of Medicine, Miami, FL, USA

2 Faculty Life Office, University of Central Florida College of Medicine, Orlando, FL, USA

3 Department of Medicine, Emory University School of Medicine, Atlanta, GA, USA

4 Department of Education, Innovation and Technology and Department of Medicine, Baylor College of Medicine, Houston, TX, USA
5 Department of Integrated Medical Science, Florida Atlantic University Charles E. Schmidt College of Medicine, Boca Raton, FL, USA

6 Office of Continuing Professional Development, Virginia Tech Carilion School of Medicine \& Carilion Clinic, Roanoke, VA, USA

7 Professional Development, Campbell University Jerry M. Wallace School of Osteopathic Medicine, Buies Creek, NC, USA

8 Office of Faculty Affairs, Virginia Commonwealth University School of Medicine, Richmond, VA, USA

9 Department of Internal Medicine, University of South Florida Morsani College of Medicine, Tampa, FL, USA 
training. Community-based clinical faculty were unable to attend on-campus sessions due to the challenges of time and distance, with faculty developers driving to satellite locations in some cases [3, 4]. Additionally, inviting speakers from other institutions or national organizations involved travel expenses and additional time for the speaker away from their work. Some medical schools offered websites with online videos and modules, but also relied heavily on in-person FD, valuing face-to-face FD for relationship building and audience interaction.

The coronavirus pandemic forced abrupt changes in medical education, requiring faculty to quickly transition student curricula online. Similarly, faculty developers pivoted to remote formats or virtual technology to provide professional development and gather faculty remotely across physical distances [5, 6]. Many faculty and faculty developers initially shared skepticism about virtual faculty development (VFD), believing that face-to-face professional development was more effective. Nevertheless, quality VFD was required. Two questions arose: how can FD be delivered virtually while maintaining quality, and would faculty participate in FD provided via a virtual platform? This article describes the authors' shared experiences in shifting to VFD activities across nine institutions.

\section{Our Experience}

The most unexpected outcome from this shift to VFD was an initial increase in the number of participants engaging in FD activities. Despite our belief that FD would not be as appealing via virtual technology, this shift actually drew in more participants than live offerings.

Across our nine institutions, as a result of the move to VFD, we drew a broader range of FD participants, particularly from satellite campuses or remote clinical education sites. Faculty noted that technology allowed them to participate without the time required for travel. Even in cities where sites were not geographically distant, faculty were able to participate more easily without the challenges of traffic or parking. All authors noted a trend of increased participation early in the pandemic, potentially due to missing in-person connecting with colleagues and communities, or due to the demands of adapting to online teaching. This was followed by a decrease to moderate levels of participation as the pandemic persisted. All were pleased to see continued increases in participation for programming targeting relevant and timely issues. For example, the number of participants attending "Educator Salons" offered by Emory University's Woodruff Health Educators Academy increased particularly for topics that were related to strategies for teaching online. Registration for one session was as high as 100 people, while pre-pandemic registration typically ranged from 20 to 50 participants. Similarly, at Florida International University
Herbert Wertheim College of Medicine, live sessions previously drew 10 to 20 faculty members; the first two sessions via Zoom drew 33 and 35 respectively. At Virginia Tech Carilion School of Medicine, the move to VFD saw a significant increase in participation, with attendance almost doubling between April and September of 2019 where 413 faculty attended, compared with 747 in 2020.

Beyond increased participation numbers, VFD had other unanticipated benefits. Faculty developers strived to engage all faculty at in-person discussions, often with a small number of faculty learners vocally participating. In line with current literature on virtual conference and seminar engagement $[7,8]$, features like chat functions of conferencing platforms allowed less vocal members of the audience to contribute, resulting in broader participation in discussions and allowing different voices to be heard.

Broader participant reach in VFD led to cross-institutional collaboration. For example, five of nine author institutions participated in a virtual medical education journal club hosted by the Southern Group on Educational Affairs (SGEA) FD Special Interest Group. Also, Virginia Commonwealth University School of Medicine extended invitations for VFD to other institutions within the SGEA. Additionally, at the University of South Florida and University of Central Florida, an increase in registration and attendance at virtual sessions was seen by members of their consortium that included academic and community institutions.

By eliminating geographic barriers, VFD programs engaged participants from multiple sites simultaneously, allowing sharing of ideas and best practices across academic sites and facilitating community building and standardization of practices across distributed clinical sites. For example, Campbell University previously brought community-based clinical faculty together annually for a clinical teaching conference on the main campus; faculty from distant sites rarely participated. VFD enabled quarterly programs for all sites, with distant faculty enthusiastically participating. This similarly occurred at the Florida International University Herbert Wertheim College of Medicine, where students train in a distributed community model across clinical sites in South Florida. A clinical VFD series was offered synchronously to all clinical faculty for the first time, supplementing the continued offerings for faculty at each clinical site. The increased efficiency of not repeating on-site programs at each location enabled a broader range of content to be shared. Overall, the virtual format has allowed for a paradigm shift from targeting individual clinical sites with FD, to instead considering FD for the entire community or academic system when developing and offering content. In the past, considering the entire academic system was a major undertaking for an on-campus event whereas with VFD, the whole system could be invited without consideration for parking, room reservation, or food. 
At in-person programs, faculty naturally interact with known colleagues. Virtual breakout rooms allowed random scattering or strategic assignment of participants to ensure interaction across diverse groupings, facilitated networking, and promoted sharing across departments or sites. For example, the SGEA journal club breakout rooms included participants from different institutions.

Faculty developers demonstrated ingenuity by mobilizing resources to do more with less during this challenging time. Six out of the nine programs invited national experts for workshops and special events with reduced costs. Hosting institution expenses for travel and hotel are eliminated with VFD as well as reducing the travel burden on expert faculty. The hosting site also incurred reduced expenses by not providing refreshments or renting space for events. Three of our nine programs were able to justify hosting nationally recognized programs of interest to faculty given the shift to a virtual format and ease of inviting experts. Some faculty developers capitalized on the opportunity by inviting other faculty from regional medical schools to participate, providing enhanced networking opportunities, and sharing costs to facilitate more opportunities in a cost-effective way.

We also saw new ideas emerge from other institutions, with some of us planning to join these collaborative efforts. Modeled after a speakers bureau, the University of North Carolina created a virtual faculty exchange to create cross-institutional connections through sharing educational knowledge [9]. Institutions who agreed to participate would commit to invite at least one speaker from another institution as part of the exchange, thus supporting the individual faculty member's professional growth while gaining outside knowledge at no cost.

Finally, the reach of FD programming grew as recording sessions increased. Five programs found that many of the faculty who were unable to attend scheduled, synchronous sessions later accessed the recordings and resources. This improved access provided a unique opportunity that will likely continue in the future. The Virginia Tech Carilion School of Medicine saw an increase in traffic on their website which houses their faculty development activities and an increase in CME credit hours claimed by faculty after viewing recorded and archived faculty development enduring materials. The Florida International University Herbert Wertheim College of Medicine created an internal repository of recorded VFD sessions on their learning management site for faculty.

\section{Applying the Lenses of Disruptive Innovation and the ARCS Framework to VFD Quality Improvement}

The concept of disruptive innovation provides a powerful lens to analyze factors essential for the success of VFD offerings. A disruptive innovation in the business world is characterized by two main principles. First, a disruptive innovation typically emerges by catering to previously overlooked consumer needs [10]. The initial success of VFD largely stemmed from its procurement of a newmarket foothold. While there were many pre-pandemic VFD opportunities, we assert that these opportunities were more readily accessible to core academic faculty but not to the thousands of clinical faculty medical schools rely on to teach students. Also, clinical faculty often prioritized their FD funding for clinical learning, which limited their participation in medical education-based programming. Lowcost opportunities successfully attracted new consumers to VFD medical education focused events.

Secondly, disruptive innovations are not attractive to all consumers until quality meets consumer criteria [10]. Certainly, the elimination of all in-person opportunities due to the pandemic likely accelerated the acceptance of VFD by consumers. With faculty developers rapidly gaining skills in designing, implementing, and evaluating VFD, the quality of VFD programs became more focused and user-friendly for all faculty. VFD removed the barriers of finding space, reserving rooms, and driving time for faculty developers, freeing them to devote energy and time towards quality virtual offerings. Further, VFD allowed faculty developers to invite outside experts, raising the quality of programs offered.

Ensuring the quality of VFD programs requires adherence to the principles of adult learning theories and other evidence-based principles in design and development. One key principle to consider is the fostering of learner motivation which plays a decisive role in online learning success. The ARCS model, introduced by Keller in 1979, highlights four motivational concepts: attention, relevance, confidence, and satisfaction [11]. This model has been widely adopted in current research and contexts to understand and construct programs to enhance learner motivation [12-16].

Learners' attention must be initially acquired and then retained for learning to occur. Instructional strategies increasing the variety of perspectives and revealing the discrepancy between different perspectives increase learner attention by arousing curiosity to learn [11]. VFD offers alternative engagement opportunities to those who struggle with new learning or worry about attention when raising ideas. We observed some faculty were more comfortable engaging by using the chat feature. Increased participant engagement increases the diversity of opinions. The convenience of VFD attracts broader participation, granting participants access to diverse perspectives and viewpoints. Busy clinicians were able to easily engage in VFD without the burden of driving or skipping lunch. The medical education literature supports reducing time and travel burdens for faculty. In one study using a virtual community of practice, authors found that online platforms for VFD allowed engagement across geographic 
boundaries [17]. Another study found that when using online CME, clinicians preferred that learning could be done when convenient, at the clinician's own pace, and at a lower cost [18].

While VFD may foster attention of learners, it may also result in new distractions for learners, removing their attention from the content and discussions. Participants may be multitasking while joining meetings by phone. Some might register for VFD only to gain access to recordings. Additional instructional design effort can enhance participants' experiences with engaging with the materials asynchronously. Faculty developers need to continue to explore creative strategies that better utilize multimedia and other features of the virtual platform to create an engaging learning environment. Future application of VFD may operate in parallel with in-person sessions requiring engagement strategies that meet the needs of both groups as part of a new post-pandemic normal.

The relevance aspect of the ARCS model refers to how the topic or the manner in which the material is taught is relevant to learners' needs and experience [11]. The high relevance of VFD in effectively addressing faculty knowledge and skill gaps, and the need to connect contributed to the initial success [19-21]. The quick transition to virtual learning while physically distancing made faculty acutely need to learn about virtual teaching, while also offering connection to colleagues at a challenging time. VFD met both needs, providing practical, immediately applicable knowledge and skills that encouraged interaction, networking, and affiliation. Buckley found that offering VFD to geographically dispersed faculty in the beginning of the pandemic, faculty highly valued connecting with each other, which allowed development of a shared, informed understanding of changes to their role, and reassurance and confidence to embrace the challenges of teaching virtually and attending to student well-being [5].

The third characteristic of the ARCS model, confidence, refers to learner's expectancy for success or failure [11]. In VFD activities, one strategy for fostering faculty confidence was to create a safe and supportive space, where faculty practice using technology in teaching. During VFD offerings, faculty acquired first-hand experience learning with new technologies and experimented with different technology tools and features with their counterparts. VFD also provided peer observation and role modeling opportunities, which further built participant confidence. For instance, at one of our sites, the faculty members who were among the first cohort to transition to online teaching subsequently led and participated in VFD sessions, sharing their best practices and lessons learned using virtual technology. This helped participants who were less familiar with online teaching to better understand what would be expected to teach with technologies and to see that online teaching is possible and achievable. The ease with which VFD is now offered and received may also contribute to the learner's confidence, including the ability to watch recordings of sessions at any time and even watching those recordings at increased speed for easy access of VFD.

The fourth aspect of the ARCS model refers to learners' satisfaction with their learning achievements, often through intrinsic or extrinsic rewards. Instructional strategies that provide opportunities of application are intrinsically rewarding [11]. Increased attendance at VFD offerings may signify increased satisfaction with this modality. The increased frequency of VFD offerings may be one example; faculty may have felt more attended to while dealing with pandemic challenges. The Florida International University Herbert Wertheim College of Medicine is considering a FD recognition program in which faculty completing VFD offerings qualify for recognition; gamifying VFD may also appeal to faculty motivation through extrinsic award.

\section{Future Directions}

Across our nine institutions, the experience of offering VFD exceeded our expectations regarding faculty participation and event quality. This experience will impact future FD, as the convenience and low cost of VFD make this format attractive and sustainable. Faculty developers have quickly acquired new technical skills and capitalized on opportunities for collaboration across institutions. While faculty developers still value face-toface learning opportunities, live sessions can be balanced with the convenience and wide availability of quality VFD in order to maximize faculty participation. Areas for future research and exploration in practice include consideration for the evaluation of efficacy of VFD and strategies to develop VFD that engage and motivate participants for virtual learning success. Focusing VFD on faculty attention, topic relevance, confidence in skills, and satisfaction with virtual formats will allow faculty developers to build on early success and maintain robust FD offerings for all faculty.

\section{Declarations}

Ethical Approval N/A.

Informed Consent N/A.

Conflict of Interest The authors declare no competing interests. 


\section{References}

1. LCME: Liaison Committee on Medical Education (LCME®). Functions and structure of a medical school. Retrieved 2 Oct 2020. https://lcme.org/wp-content/uploads/filebase/standards/2021-22_ Functions-and-Structure_2020-03-31.docx.

2. Steinert Y. Faculty development in the health professions: a focus on research and practice. Dordrecht, The Netherlands: Springer; 2014.

3. Burgess A, van Diggele C, Mellis C. Faculty development for junior health professionals. Clin Teach. 2019;16:189-96.

4. Drowos J, Baker S, Harrison SL, Minor S, Chessman AW, Baker D. Faculty development for medical school community-based faculty: a council of academic family medicine educational research alliance study exploring institutional requirements and challenges. Acad Med. 2017;92:1175-80.

5. Buckley H. Faculty development in the COVID-19 pandemic: so close - yet so far. Med Educ. 2020;20. https://doi.org/10.1111/ medu. 14250 .

6. Chertoff JD, Zarzour JG, Morgan DE, Lewis PJ, Canon CL, Harvey JA. The early influence and effects of the coronavirus disease 2019 (COVID-19) pandemic on resident education and adaptations. J Am Coll Radiol. 2020;17:1322-8.

7. Vervoort D, Dearani JA, Starnes VA, Thourani VH, Nguyen TC. Brave new world: virtual conferencing and surgical education in the coronavirus disease 2019 era. J Thorac Cardiovasc Surg. 2021;161:748-52.

8. Carrillo C, Flores MA. COVID-19 and teacher education: a literature review of online teaching and learning practices. Eur J Teach Educ. 2020;43:466-87.

9. Academies Collaborative Newsletter. Fall. 2020. Retrieved December 5, 2020. https://www.academiescollaborative.com/ fall-2020-newsletter.htm.

10. Christensen CM, Raynor ME, McDonald R. What is disruptive innovation. Harv Bus Rev. 2015;93:44-53.

11. Keller J. Development and use of the ARCS model of instructional design. J Instr Dev. 1987;10:2-10.
12. Li K, Keller JM. Use of the ARCS model in education: a literature review. Comput Educ. 2018;122:54-62.

13. Milman NB, Wessmiller J. Motivating the online learner using Keller's ARCS model. Distance Learning. 2016;13:67-71.

14. Pinchevsky-Font T, Dunbar S. Best practices for online teaching and learning in health care related programs. Internet J Allied Health Sci Practice [Internet]. 2015;13. Available from: https:// nsuworks.nova.edu/ijahsp/vol13/iss1/8. Accessed 4 Jan 2022.

15. Blair C, Walsh C, Best P. Immersive $360^{\circ}$ videos in health and social care education: a scoping review. BMC Med Educ. 2021;21:1-28.

16. Shell K, Holt E, Kington A, Mohammed K, Black A, Troup C, Ingiaimo M, Scoles K, Nathaniel TI. Motivation to learn neuroanatomy by cadaveric dissection is correlated with academic performance. Clin Anat. 2020;33:128-35.

17. Chan TM, Gottlieb M, Sherbino J, Cooney R, Boysen-Osborn M, Swaminathan A, Ankel F, Yarris LM. The ALiEM faculty incubator: a novel online approach to faculty development in education scholarship. Acad Med. 2018;93:1497-502.

18. Pott MO, Blanshan AS, Huneke KMMS, Baasch Thomas BL, Cook DA. What influences choice of continuing medical education modalities and providers? A national survey of U.S. physicians, nurse practitioners, and physician assistants. Acad Med. 2021;96:93-100.

19. Hollebrands KF, Lee HS. Effective design of massive open online courses for mathematics teachers to support their professional learning. ZDM. 2020;52:859-75.

20. Qian Y, Hambrusch S, Yadav A, Gretter S. Who needs what: recommendations for designing effective online professional development for computer science teachers. J Res Technol Educ. 2018;50:164-81.

21. Powell CG, Bodur Y. Teachers' perceptions of an online professional development experience: implications for a design and implementation framework. Teach Teach Educ. 2019;77:19-30.

Publisher's Note Springer Nature remains neutral with regard to jurisdictional claims in published maps and institutional affiliations. 